\title{
Relaxation in a glassy binary mixture: Mode-coupling-like power laws, dynamic heterogeneity and a new non-Gaussian parameter
}

\author{
Elijah Flenner and Grzegorz Szamel \\ Department of Chemistry, Colorado State University, Fort Collins, CO 80525
}

(Dated: August 20, 2018)

\begin{abstract}
We examine the relaxation of the Kob-Andersen Lennard-Jones binary mixture using Brownian dynamics computer simulations. We find that in accordance with mode-coupling theory the self-diffusion coefficient and the relaxation time show power-law dependence on temperature. However, different mode-coupling temperatures and power laws can be obtained from the simulation data depending on the range of temperatures chosen for the power-law fits. The temperature that is commonly reported as this system's mode-coupling transition temperature, in addition to being obtained from a power law fit, is a crossover temperature at which there is a change in the dynamics from the high temperature homogeneous, diffusive relaxation to a heterogeneous, hopping-like motion. The hopping-like motion is evident in the probability distributions of the logarithm of single-particle displacements: approaching the commonly reported mode-coupling temperature these distributions start exhibiting two peaks. Notably, the temperature at which the hopping-like motion appears for the smaller particles is slightly higher than that at which the hopping-like motion appears for the larger ones. We define and calculate a new non-Gaussian parameter whose maximum occurs approximately at the time at which the two peaks in the probability distribution of the logarithm of displacements are most evident.
\end{abstract}

PACS numbers: $61.20 \mathrm{Lc}, 64.70 \mathrm{Pf}, 61.43$ Fs

\section{INTRODUCTION}

Computer simulations have added a great deal to our knowledge of relaxation in supercooled liquids. A seminal series of studies was performed by Kob and Andersen [1] who were interested in comparing the results of molecular dynamics simulations to the predictions of the modecoupling theory [2]. They observed that as the temperature was lowered the mode-coupling theory gave a good qualitative description of the relaxation of the liquid. Specifically, they found that the long-time self-diffusion coefficient and a characteristic relaxation time showed power law behavior as $T_{c}=0.435$ was approached. Since their investigation, this temperature has been referred to as the mode-coupling transition temperature for the Kob-Andersen Lennard-Jones mixture. Later work 3, 4] demonstrated that there is no true vanishing of the selfdiffusion coefficient or true divergence of the $\alpha$ relaxation time at $T_{c}$. This is similar to what is observed experimentally for most glass formers 5]. There exists power law like behavior of the relaxation time as a temperature $T_{c}$ is approached and close to $T_{c}$ there is a crossover to a different relaxation scenario which extends to the laboratory glass transition $T_{g}$, defined as the temperature at which the viscosity equals $10^{13}$ poise [5, 6, 7].

Many simulations performed since the Kob and Andersen study have resulted in the following relaxation scenario in supercooled liquids around the crossover temperature $T_{c}$ (see Ref. 10] and references therein). The motion of particles in a slightly supercooled liquid is homogeneous and the self part of the van Hove correlation function [8] is approximately Gaussian at all times. At lower temperatures the self part of the van Hove correlation function deviates significantly from Gaussian, the motion of the particles is strongly heterogeneous, and, on an intermediate time scale (up to so-called exchange time), the particles can be separated by their individual relaxation times. It is generally believed that at short times the particles are confined to cages of neighboring particles and a "cooperative motion" 11] of particles has to occur to facilitate cage escape [12, 13. The timescale of this cooperative motion increases with decreasing temperature. In the original studies [11, 12] it was assumed that this time scale is around the peak in the non-Gaussian parameter $\alpha_{2}(t)=\frac{3}{5}\left\langle\delta r^{4}\right\rangle /\left\langle\delta r^{2}\right\rangle^{2}-1$, where $\delta r$ is the distance over which a particle moved in time $t$. It has been accepted that the peak position of $\alpha_{2}(t)$ "roughly locates the time of maximum dynamic heterogeneity" 14. While this is a natural first choice, there is no a priori reason to choose this time. It has been shown that the van Hove correlation function deviates strongly from a Gaussian distribution over a much longer time scale [15]. Moreover, it is also possible to identify particles which remain slower than other particles for time much longer than the time scale given by the peak position of $\alpha_{2}(t)[16]$. We should point out that, although the time scale of the peak position of $\alpha_{2}(t)$ is often singled out in connection with dynamic heterogeneity, it has also been recognized that there exists dynamic heterogeneity on a much longer time scale, and a four-point correlation function that is sensitive to this longer-time heterogeneity has been defined and investigated (see Ref. [17] and references therein).

In this work we examine the relaxation of a supercooled liquid using Brownian dynamics computer simulations. Experiments conducted on Brownian systems (i.e. on colloidal suspensions) have so far failed to show any significant departure from the power law behavior of 
the self-diffusion coefficient and the characteristic relaxation time 18]. Thus, it was suggested that the predictions of the original (also known as "idealized") modecoupling theory provide a good description of the relaxation of the fluid. However, it has since been demonstrated using computer simulations that the relaxation of a supercooled fluid is the same for Newtonian dynamics 1], stochastic dynamics [4], and Brownian dynamics 15]. In particular, in computer simulations departures from mode-coupling-like power laws and emergence of hopping-like motion have been observed for all three microscopic dynamics. The qualitative difference between experimental results (which do not find deviations from mode-coupling-like power laws) and theoretical ones (which do) remains unexplained.

The focus of this work is the crossover from the high temperature homogeneous relaxation of the slightly supercooled fluid to the low temperature relaxation around $T_{c}$. We show that, while the mode-coupling transition temperature cannot be unambiguously determined from the computer simulation results, the commonly reported temperature is the crossover temperature for two different modes of relaxation. Furthermore, we define and calculate a time-dependent function that vanishes identically for a Gaussian diffusion process. This new function $\gamma(t)=\frac{1}{3}\left\langle\delta r^{2}\right\rangle\left\langle 1 / \delta r^{2}\right\rangle-1$, hereafter called a new nonGaussian parameter, has a peak at a time at which the relaxation seems the most heterogeneous. This time is always longer than a characteristic decay time of the incoherent intermediate scattering function [9] that is known as the $\alpha$ relaxation time. Moreover, the peak position of the new non-Gaussian parameter has temperature dependence very similar to that of the $\alpha$ relaxation time.

The paper is organized as follows. In section III we briefly describe the simulation. In section III we present results for the mean square displacement, the selfdiffusion coefficient, the incoherent intermediate scattering functions, and the $\alpha$ relaxation time (a preliminary report of some of these results appeared in Ref. [15]). We show that two different power-law fits can be obtained depending on the range of temperatures used. In section IV we present results for the probability distributions of the logarithm of single-particle displacements. These distributions are sensitive to hopping-like dynamics. In section $\nabla$ we define and present the new non-Gaussian parameter. In section $\nabla \mathbb{1}$ we discuss the conclusions that can be drawn from this work.

\section{SIMULATION DETAILS}

We simulated a binary mixture of 800 particles of type A and 200 particles of type B that was first considered by Kob and Andersen [1]. Briefly, the interaction potential is $V_{\alpha \beta}(r)=4 \epsilon_{\alpha \beta}\left[\left(\sigma_{\alpha \beta} / r\right)^{12}-\left(\sigma_{\alpha \beta} / r\right)^{6}\right]$, where $\alpha, \beta \in\{A, B\}, \epsilon_{A A}=1.0, \sigma_{A A}=1.0, \epsilon_{A B}=1.5$, $\sigma_{A B}=0.8, \epsilon_{B B}=0.5$, and $\sigma_{B B}=0.88$. The interaction parameters are chosen to prevent crystallization [1].
The simulations are performed with the interaction potential cut at $2.5 \sigma_{\alpha \beta}$, and the box length of the cubic simulation cell is $9.4 \sigma_{A A}$. Periodic boundary conditions were used.

We performed Brownian dynamics simulations. The equation of motion for the position of the $i_{t h}$ particle of type $\alpha, \vec{r}_{i}^{\alpha}$, is

$$
\dot{\vec{r}}_{i}^{\alpha}=\frac{1}{\xi_{0}} \vec{F}_{i}^{\alpha}+\vec{\eta}_{i}(t)
$$

where the friction coefficient of an isolated particle $\xi_{0}=$ 1.0 and $\vec{F}_{i}^{\alpha}$ is the force acting on the $i_{t h}$ particle of type $\alpha$,

$$
\vec{F}_{i}^{\alpha}=-\nabla_{i}^{\alpha} \sum_{j \neq i} \sum_{\beta=1}^{2} V_{\alpha \beta}\left(\left|\vec{r}_{i}^{\alpha}-\vec{r}_{j}^{\beta}\right|\right)
$$

with $\nabla_{i}^{\alpha}$ being the gradient operator with respect to $\vec{r}_{i}^{\alpha}$. In Eq. (11) the dot denotes a time derivative, and the random noise $\vec{\eta}_{i}$ satisfies the fluctuation-dissipation theorem,

$$
\left\langle\vec{\eta}_{i}(t) \vec{\eta}_{j}\left(t^{\prime}\right)\right\rangle=2 D_{0} \delta\left(t-t^{\prime}\right) \delta_{i j} \mathbf{1} .
$$

In Eq. (31), the diffusion coefficient $D_{0}=k_{B} T / \xi_{0}$ where $k_{B}$ is Boltzmann's constant and $\mathbf{1}$ is the unit tensor. Since the equation of motion allows for diffusive motion of the center of mass, all the results will be presented relative the center of mass (i.e. momentary positions of all the particles are always relative to the momentary position of the center of mass). We will present the results in terms of the reduced units with $\sigma_{A A}, \epsilon_{A A}, \epsilon_{A A} / k_{B}$, and $\sigma_{A A}^{2} \xi_{0} / \epsilon_{A A}$ being the units of length, energy, temperature, and time, respectively. The mass of both particles are the same and equal to 1.0.

The equations of motion, Eq. 1] were solved using a Heun algorithm with a small time step of $5 \times 10^{-5}$. We simulated the temperatures $T=0.44,0.45,0.47,0.5$, $0.55,0.6,0.8,0.9,1.0,2.0,3.0$, and 5.0. We ran a long equilibration run (at least half as long as the production run) and four production runs at each temperature, except at $T=0.44$ where we ran six production runs. The results are an average over the production runs, which were as long as $6 \times 10^{8}$ steps long for the lowest temperature studied.

\section{MODE-COUPLING-LIKE POWER LAWS}

The mode-coupling theory predicts a power law vanishing of the self-diffusion coefficient and a power law divergence of the characteristic relaxation time at the mode-coupling transition temperature $T_{c}$. In many simulations and experiments there is a range of temperatures where power laws fit the diffusion coefficient and the relaxation time well, and the transition temperature is obtained from fits of these properties to functions of the 


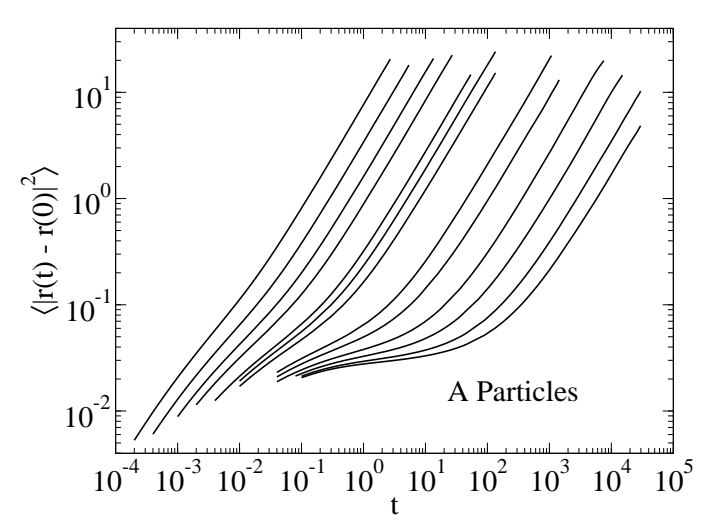

FIG. 1: The mean square displacement for the A particles for $T=5.0,3.0,2.0,1.5,1.0,0.9,0.8,0.6,0.55,0.50,0.47,0.45$, 0.44 listed from left to right. The graph of the mean square displacement for the B particles is similar.

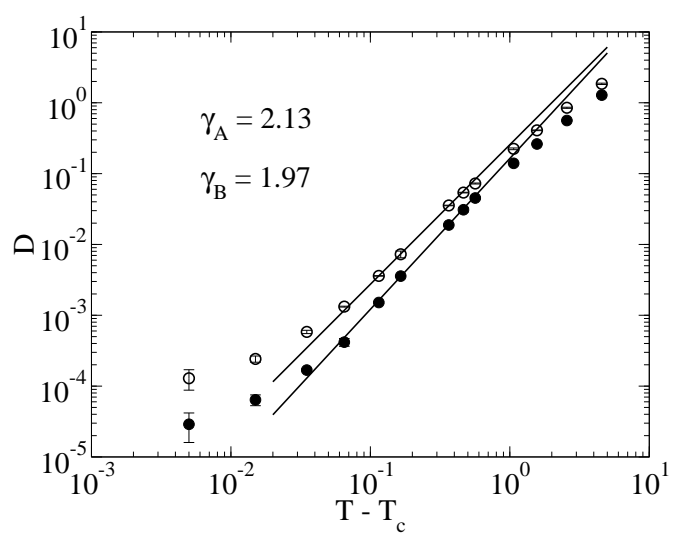

FIG. 2: Long time diffusion coefficient for the A (closed symbols) and the B (open symbols) particles. The lines are the power law fits to the function $a\left(T-T_{c}\right)^{\gamma}$ with $T_{c}=0.435$ fixed.

form $a\left(T-T_{c}\right)^{\gamma}$. The $T_{c}$ obtained in this manner is generally referred to as the mode-coupling temperature. In this section, we will present results for the mean square displacement, the diffusion coefficient, the self intermediate scattering functions and the $\alpha$ relaxation time. Moreover, we will show that for the system studied in this work reasonable power law fits can be obtained for the diffusion coefficient and the $\alpha$ relaxation time for a transition temperature different from the usually accepted mode-coupling transition temperature of $T_{c}=0.435$ if a different range of temperatures is used for the power law fits.

Shown in Fig. 1 is the single-particle mean square displacement $\left\langle\delta r^{2}(t)\right\rangle=\left\langle\left|\vec{r}^{A}(t)-\vec{r}^{A}(0)\right|^{2}\right\rangle$ for the A particles. The graph for the B particles looks similar. The short time motion is diffusive with a temperature dependent diffusion coefficient $D_{0}=k_{B} T / \xi_{0}$ where $k_{B}$ is Boltzmann's constant and $\xi_{0}$ is the friction coefficient (recall that in our units $k_{B}=1$ and $\xi_{0}=1$ ). For low temperatures a plateau develops in the log-log plot at

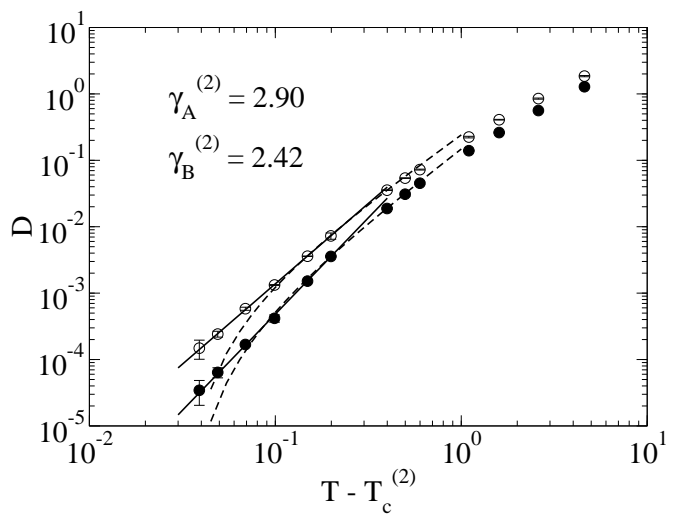

FIG. 3: Diffusion coefficient for the A (closed symbols) and B (open symbols) particles. The solid lines are the "alternative" power-law fits to the function $a^{(2)}\left(T-T_{c}^{(2)}\right)^{\gamma^{(2)}}$ where $T_{c}^{(2)}=$ 0.401 . Dashed lines are fits to the function $a\left(T-T_{c}\right)^{\gamma}$ where $T_{c}=0.435$.

intermediate times where the mean square displacement does not change appreciably. For the lowest temperature the plateau region spans several decades in time.

The plateau region is generally associated with the "cage" effect. It represents a localization of the particles on intermediate time scales and has been observed in many simulations of glassy systems [1, 3]. Note that there is no true plateau in the mean square displacement versus time and the slope of the mean square displacement versus time decreases monotonically. Therefore there is no inflection point of the mean square displacement as a function of time. However, there is an inflection point in the logarithm of the mean square displacement versus the logarithm of time. We use this inflection point to find the cage diameter. For $T=0.44$ the inflection point occurs at a value of the mean square displacement of 0.0288 $\sigma_{A A}$ for the A particles and $0.0461 \sigma_{A A}$ for the B particles. These values of the cage diameter corresponds to a distance around $0.17 \sigma_{A A}$ for the A particles and around $0.21 \sigma_{A A}$ for the B particles, which is much smaller than the diameter of any particle. After the plateau, the motion is again diffusive with a diffusion coefficient $D<D_{0}$.

We determined the long-time self-diffusion coefficient $D$ from the slope of $\left\langle\delta r^{2}(t)\right\rangle$ at long times. The results for the $A$ and $B$ particles are shown in figure 2 We observe power law behavior similar to what was reported in previous simulations of this system using Newtonian [1] and stochastic dynamics [3, 4]. Namely, there is power law behavior of the diffusion coefficient for temperatures between $T=0.8$ and $T=0.50$. Then there are deviations from the power law at and below $T=0.47$. We fit the diffusion coefficients for $0.5 \leq T \leq 0.8$ for the $A$ and $B$ particles to a power law of the form $a(T-0.435)^{\gamma}$. The exponents in the fit are given in the figure, and agree reasonably well with the exponents found using Newtonian [1] and stochastic dynamics [3, 4, 19].

We also performed three parameter fits to the diffusion coefficient and the $\alpha$ relaxation time for different temper- 


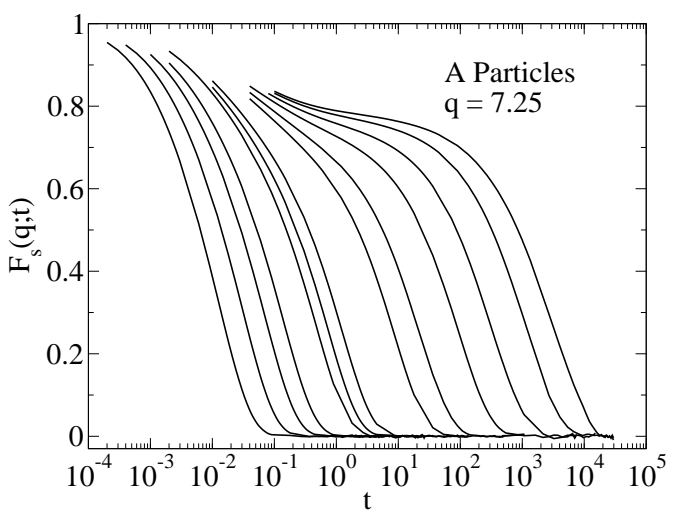

FIG. 4: Self intermediate scattering functions for the A particles for $T=5.0,3.0,2.0,1.5,1.0,0.90,0.80,0.60,0.55,0.50$, $0.47,0.45$, and 0.44 listed from left to right. The corresponding graph for the B particles is similar.

ature ranges [20]. We found that we can obtain good fits to $D$ and $\tau_{\alpha}$ for temperatures $0.44 \leq T \leq 0.6$. The transition temperature depends slightly on the quantity being fitted, and ranges from $T_{c}=0.391$ to $T_{c}=0.409$. We found the average transition temperature obtained from the fits $T_{c}^{(2)}=0.401 \pm 0.009$, and then fit each quantity to the function $a^{(2)}(T-0.401)^{\gamma^{(2)}}$. We show this final fit for the self-diffusion coefficient in Fig. 3. Qualitatively, it is clear that the new fit is as good as the standard fit shown in Fig. 2] Quantitatively, we evaluate the quality of fit by examining $\chi^{2}=\sum_{i}^{N}\left[\left(D\left(T_{i}\right)-y\left(T_{i}\right)\right) / \sigma_{i}\right]^{2}$ where $N$ is the number of data points used in the fit, $y(T)=a\left(T-T_{c}\right)^{\gamma}$ and $\sigma_{i}$ is the standard deviation of the diffusion coefficient at $T_{i}$. For each fit we find the probability $p$ that the value of $\chi^{2}$ should exceed the calculated value by chance given that the model is correct 21. Higher values of $p$ correspond to a better fit, and the maximum value of $p$ is one. For the transition temperature $T_{c}^{(2)}=0.401$ and the temperature range $0.44 \leq T \leq 0.6$, $p=0.860$ for the A particles and $p=0.902$ for the B particles. This is significantly better than the values $p=0.044$ and $p=0.0169$ for the A and B particles, respectively, when the diffusion coefficient was fit over the temperature range $0.5 \leq T \leq 0.8$ and with $T_{c}=0.435$.

We calculated the self intermediate scattering function $F_{s}^{\alpha}(q, t)=\frac{1}{N_{\alpha}}\left\langle\sum_{j=1}^{N_{\alpha}} \exp \left[i \vec{q} \cdot\left(\vec{r}_{j}^{\alpha}(t)-\vec{r}_{j}^{\alpha}(0)\right)\right]\right\rangle, \alpha \in$ $\{A, B\}$, shown in Fig. 4 for a wave vector $q=|\vec{q}|$ around the first peak of the partial structure factors for the A $(q=7.25)$ and the B $(q=5.75)$ particles. The self intermediate scattering function decays from its $t=0$ value of one to zero. For the same temperatures in which there is a plateau in the log-log plot of the mean square displacement, there is also a plateau in the log-log plot of the incoherent intermediate scattering functions. The characteristic time for the decay of the self intermediate scattering function is the $\alpha$ relaxation time $\tau_{\alpha}$, which we define as the time when this scattering function is equal to $1 / e$ of its initial value. It has been observed that other

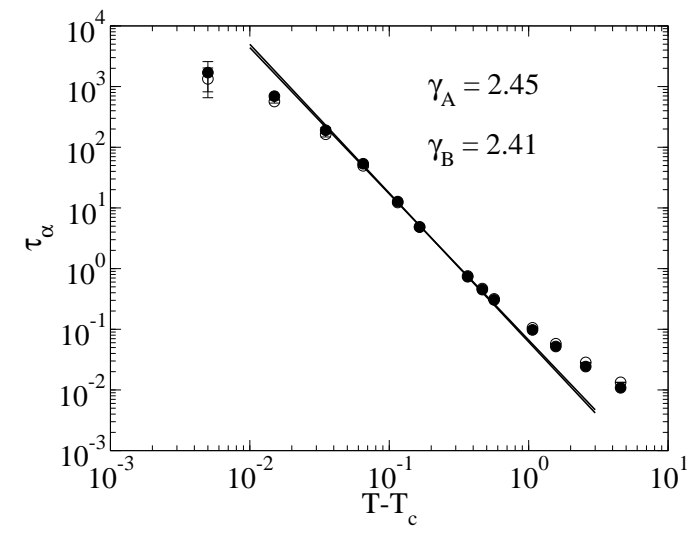

FIG. 5: The $\alpha$ relaxation time for the A (closed symbols) and $\mathrm{B}$ (open symbols) particles. The lines are fits to the function $a\left(T-T_{c}\right)^{-\gamma}$ with $T_{c}=0.435$ fixed.

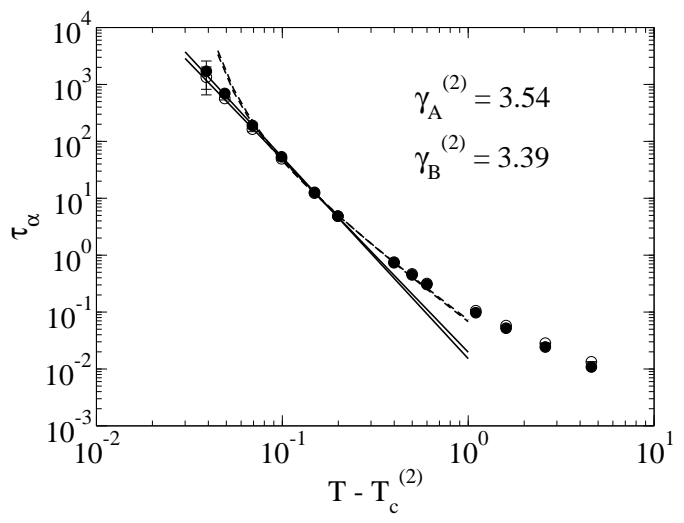

FIG. 6: The $\alpha$ relaxation time for the A (closed symbols) and B (open symbols) particles. The solid line are the "alternative" power-law fits to the function $a^{(2)}\left(T-T_{c}^{(2)}\right)^{-\gamma^{(2)}}$ where $T_{c}^{(2)}=0.401$. Dashed lines are fits to the functions $a\left(T-T_{c}\right)^{-\gamma}$ where $T_{c}=0.435$.

definitions of the $\alpha$ relaxation time results in the same temperature dependence.

The $\alpha$ relaxation time is shown in Fig. 5 Again there is power law behavior of the $\alpha$ relaxation time between $0.5 \leq T \leq 0.8$, then there are deviations from the power law for temperatures at and below $T=0.47$. The lines in the figure are power law fits to the function $a(T-$ $0.435)^{-\gamma}$ for the temperature range $0.5 \leq T \leq 0.8$, which is the same function and the same temperature range as for Fig. 2 The exponents are given in Fig. 5 and are close to what was found in simulations of the same system using Newtonian [1] and stochastic dynamics [3, 4] instead of Brownian dynamics.

As explained above, we also fit the $\alpha$ relaxation time to the function $a^{(2)}(T-0.401)^{-\gamma^{(2)}}$. The result is shown in Fig. [6] Again, qualitatively, the new fit is as good as the standard fit shown in Fig. [5. Quantitatively, we evaluated the quality of fits using the same procedure as for the diffusion coefficient. When $T_{c}$ was set to 0.401 and we fit 
the $\alpha$ relaxation time for $0.44 \leq T \leq 0.6, p=0.598$ for the A particles and $p=0.743$ for the B particles. When the transition temperature was set to $T_{c}=0.435$ and we used the temperature range $0.5 \leq T \leq 0.8, p=0.15$ for the A particles and $p=0.35$ for the $\mathrm{B}$ particles. Thus the fits were better for $T_{c}^{(2)}=0.401$.

The values of the scaling exponents obtained from new fits for both the self-diffusion coefficient and the $\alpha$ relaxation time are quite a bit greater than those obtained from the conventional fit. Also, the difference between the exponents for the $\mathrm{A}$ and $\mathrm{B}$ particles is considerably larger.

We should point out that one could try using a slightly different procedure to identify the mode-coupling transition temperature. Namely, one could try fit a straight line to a plot of, e.g $D^{1 / \gamma}$ vs. temperature, where $\gamma$ is a scaling exponent obtained from solving mode-coupling equations. The are two potentials problems with this approach: first, it requires solving full time and wave-vector dependent mode-coupling equations [22]. Second, using this alternative approach one has to neglect the qualitative differences between the predictions of the theory and results of the simulations like, e.g. the difference between scaling exponents for the $\mathrm{A}$ and the $\mathrm{B}$ particles and the difference between scaling exponents for the diffusion coefficients and the relaxation times.

In conclusion, we find that the mode-coupling temperature of the Kob-Andersen binary mixture is not unique: if a temperature range different from the original one 1 is used for fitting the simulation data to the power laws, a different mode-coupling transition temperature results. In the next section we show that there is a change in the dynamics around the commonly reported mode-coupling temperature, $T_{c}=0.435$.

\section{PROBABILITY DISTRIBUTIONS OF SINGLE PARTICLE DISPLACEMENTS}

Following a procedure suggested previously [14, 23, 24], we examined the probability distributions of the logarithm of single-particle displacements $P\left(\log _{10}(\delta r) ; t\right)$ at a time $t$. Multiple peaks in $P\left(\log _{10}(\delta r) ; t\right)$ at a time $t^{\prime}$ have been clearly observed in simulations of model colloidal gels [14], and provide evidence of populations of fast and slow particles at $t^{\prime}$. Note that $P\left(\log _{10}(\delta r) ; t\right)$ is defined such that the integral $\int_{x_{0}}^{x_{1}} P(x ; t) \mathrm{d} x$ is the fraction of particles whose value of $\log _{10}(\delta r)$ is between $x_{0}$ and $x_{1}$. Furthermore, the probability distribution $P\left(\log _{10}(\delta r) ; t\right)$ can be obtained from the self van Hove correlation function [9] since $P\left(\log _{10}(\delta r) ; t\right)=\ln (10) 4 \pi \delta r^{3} G_{s}(\delta r, t)$. There are some general properties of $P\left(\log _{10}(\delta r) ; t\right)$ if the van Hove correlation function is Gaussian. If the motion of a tagged particle is diffusive at all times with a diffusion coefficient $D$, then the self van Hove correlation function $G_{s}(\delta r, t)=\left(1 /(4 \pi D t)^{\frac{3}{2}}\right) \exp \left(-\delta r^{2} / 4 D t\right)$. For a Gaussian van Hove function, the shape of $P\left(\log _{10}(\delta r) ; t\right)$ is independent of time [25], and the peak height of
$P\left(\log _{10}(\delta r) ; t\right)=\log _{e}(10) \sqrt{54 / \pi} e^{3 / 2} \approx 2.13$. Deviations from this height represents deviations from Gaussian behavior of $G_{s}(r, t)$.

Shown in Fig. 7 is $P\left(\log _{10}(\delta r) ; t\right)$ for the A particles at $T=5.0,0.6,0.47$, and 0.44 . For each temperature several different times are shown. The thicker lines correspond to later times. At $T=5.0$ there is little deviation from Gaussian behavior: there is only one peak whose height is close to 2.13 at all times. The peak position moves to larger distances for larger times. For $T=0.6$, there are deviations from Gaussian behavior manifested in the reduced height of the peak of the distribution, but there is still only one peak for all times. At $T=0.47$, the deviations from Gaussian behavior are stronger and the distribution becomes very broad at a time which corresponds to right after the plateau of the mean square displacement. At $T=0.44$ there are two distinct peaks. The position of the second peak depends on time, but when the height of both peaks are approximately equal the position of the second peak is around $\log _{10}(\delta r)=0$, thus $\delta r=1.0=\sigma_{A A}$.

The probability distributions $P\left(\log _{10}(\delta r) ; t\right)$ provide clear evidence that there are populations of particles with different mobilities. These probability distributions are similar to the distributions observed in model colloidal gels close to a gelation transition [23] and in dense systems with purely repulsive interactions [14]. The shape of the distributions strongly suggests a heterogeneous hopping-like motion for at least a fraction of the particles. We find that the hopping rate of the particles varies greatly between particles, and the typical size of the hopping length for the A (larger) particles is equal to one particle diameter.

The two peaks in $P\left(\log _{10}(\delta r) ; t\right)$ are clearly defined at a higher temperature for the B particles, Fig. 8 than for the A particles. For $T=5.0, P\left(\log _{10}(\delta r) ; t\right)$ for the $\mathrm{B}$ particles is similar to what is observed for the A particles. The distribution $P\left(\log _{10}(\delta r) ; t\right)$ broadens and the peak height decreases significantly for the B particles starting at $T=0.6$, and we observe the two peak structure starting at $T=0.5$. The two peaks are well defined for $T=0.47$ and are very prominent for $T=0.44$. At these low temperatures there is a clear distinction between mobile and immobile particles. The second peak occurs around $\log _{10}(\delta r) \approx 0.086$ for the B particles, which corresponds to a value of $\delta r \approx 1.25$. This suggests that the typical length of the particle jumps are slightly larger for the B particles than for the A particles. Note that there are small secondary peaks in $P\left(\log _{10}(\delta r) ; t\right)$ for the $\mathrm{B}$ particles for the longer times. This is expected if the activated hopping is the dominant relaxation process.

The size dependence of the distributions of the displacements of the particles have been observed in simulations of colloidal gels by Puertas et al. 23]. They simulated a system of soft core polydisperse particles with an average particle radius $a$. They noticed that the distributions of squared displacements were more bimodal for particles with a smaller radius (see Fig. 6 in Ref. 23] ). It 

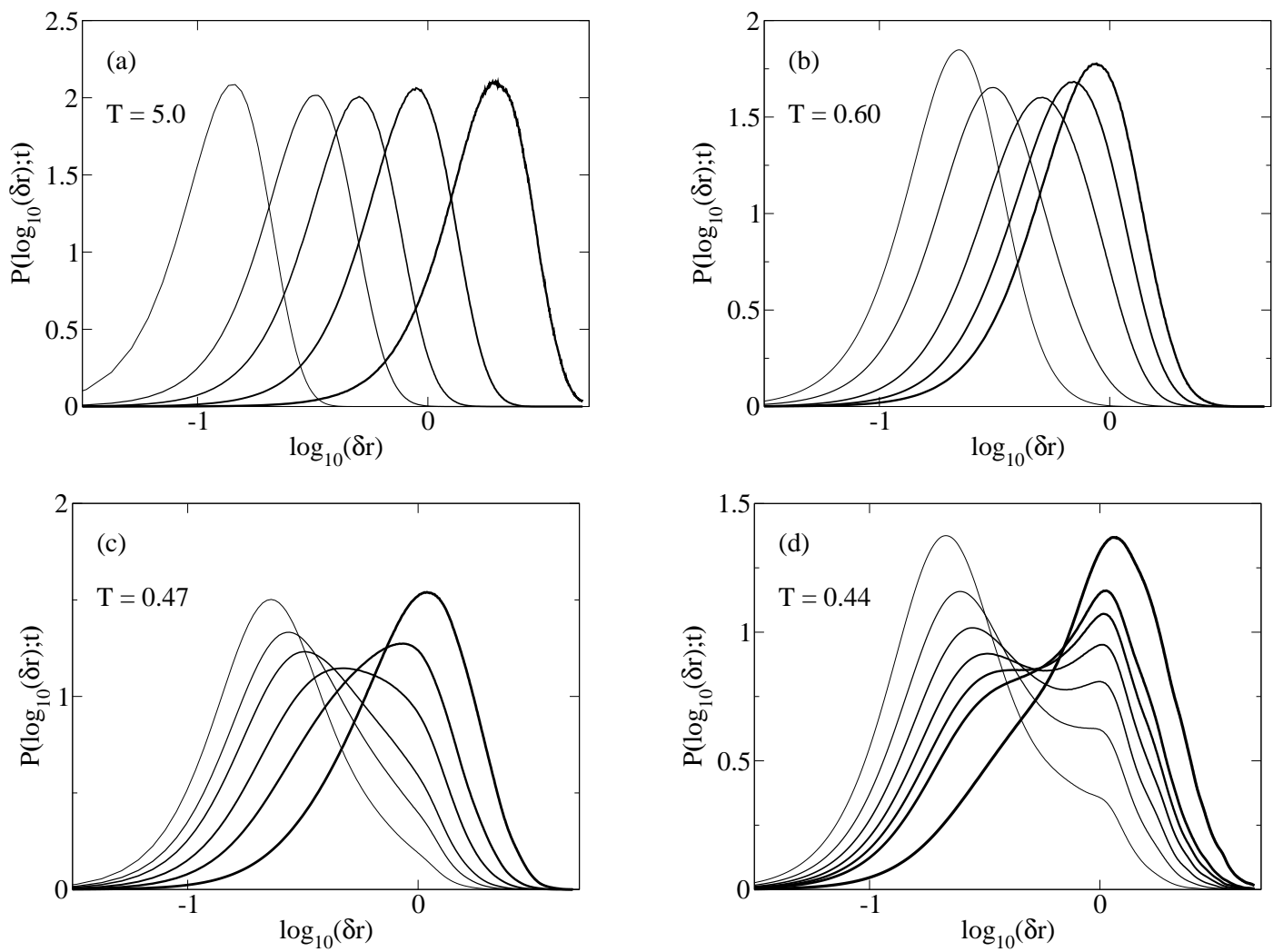

FIG. 7: The probability of the logarithm of single particle displacements $P\left(\log _{10}(\delta r)\right.$; $\left.t\right)$ for the A particles. The wider lines indicate increased time $t$. a: $T=5.0$ (a) for $t=0.01,0.05,0.1,5$ and 10 listed from left to right. b: $T=0.60$ for $t=1.0,5,15$, 25 and 30 listed from left to right. c: $T=0.47$ for $t=100,200,300,400,500$, and 800 listed from left to right. d: $T=0.44$ for $t=1000,2000,3000,4000,5000,6000$, and 10000 listed from left to right.

is likely that the size dependence of dynamic heterogeneity is a general feature of systems with slow dynamics.

In earlier studies [14, 23] probability distributions of particle displacements were examined at a time $t^{*}$ such that $\left\langle\delta r^{2}\left(t^{*}\right)\right\rangle=10 a^{2}$, where $a$ is a measure of the average size of the polydisperse particles. While bimodal distributions were observed for this $t^{*}$, there was no clear justification for selecting this particular timescale. In the next section we introduce a new non-Gaussian parameter whose peak position allows us to identify the time in which the two peaks in $P\left(\log _{10}(\delta r) ; t\right)$ are around the same height. Moreover, we will demonstrate that the peak position of the commonly used non-Gaussian parameter $\alpha_{2}(t)$ does a poor job of identifying this time.

\section{NEW NON-GAUSSIAN PARAMETER}

Many simulations which examine heterogeneous dynamics focus on a timescale which is found from the peak of the non-Gaussian parameter $\alpha_{2}(t)=$ $\frac{3}{5}\left\langle\delta r^{4}(t)\right\rangle /\left\langle\delta r^{2}(t)\right\rangle^{2}-1$. It has been observed that close to $T_{c}=0.435, \alpha_{2}(t)$ has a peak occurring at the socalled late $\beta$ regime, i.e. some time before the $\alpha$ relaxation time. Moreover, for $T<0.8$ with decreasing tem- perature the value of the non-Gaussian parameter at the $\alpha$ relaxation time, $\alpha_{2}\left(\tau_{\alpha}\right)$, is a decreasing fraction of its maximum value [15. In particular, at $T=0.44$ the nonGaussian parameter at the $\alpha$ relaxation time is approximately equal to 0.62 and 0.35 of its maximum value for the $\mathrm{A}$ and $\mathrm{B}$ particles, respectively. We observe that the two peaks in $P\left(\log _{10}(\delta r) ; t\right)$ (for temperatures in which the two peaks are clearly defined) are about the same height at some time after the $\alpha$ relaxation time. Thus $\alpha_{2}(t)$ is small compared to its maximum value at a time when the two peaks are very prominent.

We propose to use a new function that, as does $\alpha_{2}(t)$, quantifies deviations from a Gaussian distribution of displacements,

$$
\gamma(t)=\frac{1}{3}\left\langle\delta r^{2}\right\rangle\left\langle\frac{1}{\delta r^{2}}\right\rangle-1,
$$

where $\delta r$ is the distance over which the particle moved in time $t$. We will show that this new non-Gaussian parameter identifies the time in which the two peaks in the probability distribution of the logarithm of single-particle displacements are most evident. The parameter $\alpha_{2}(t)$ is significantly influenced mostly by particles which move farther than expected from a Gaussian distribution of particle displacements. In contrast, the parameter $\gamma(t)$ 

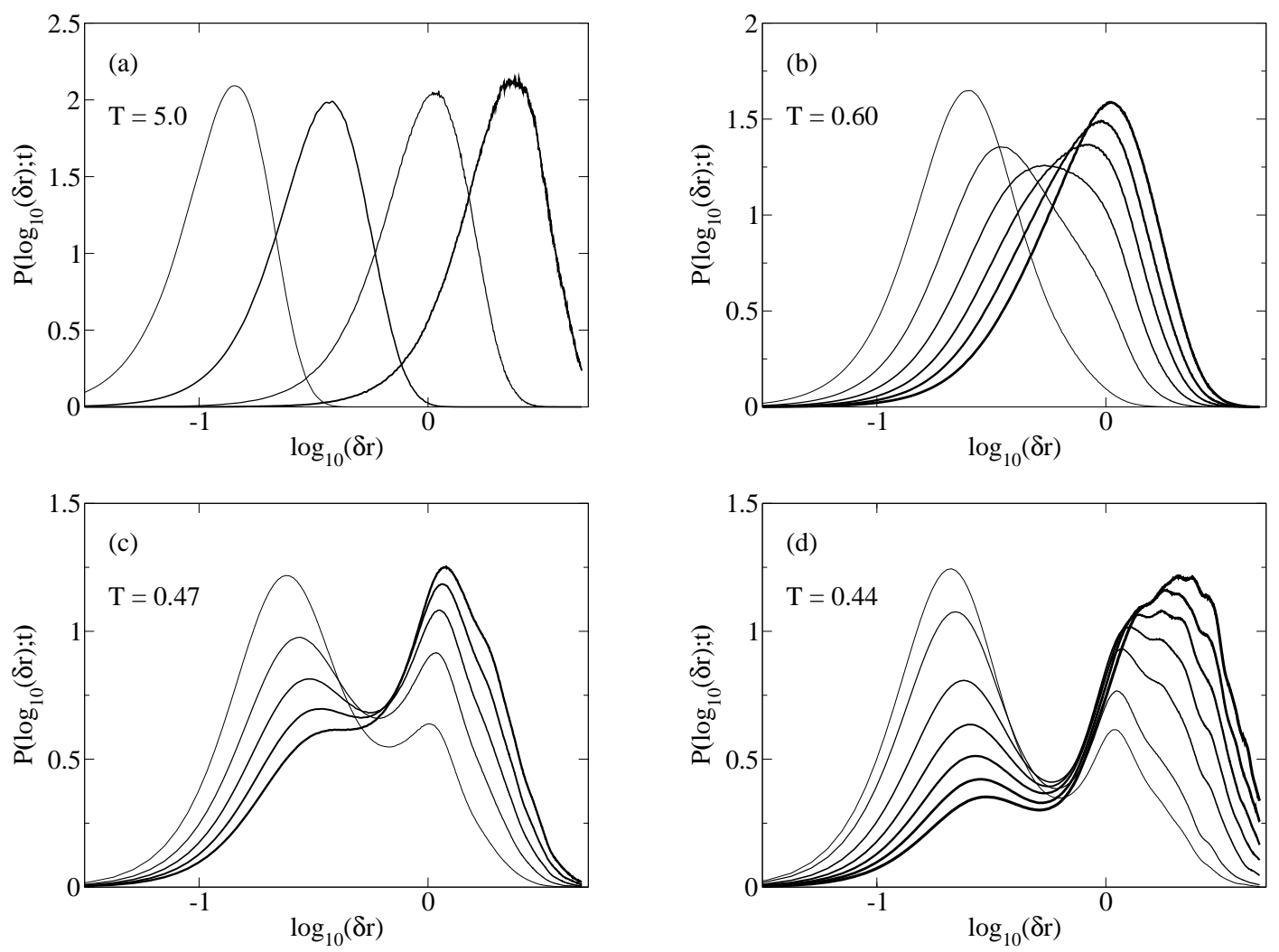

FIG. 8: The probability of the logarithm of single particle displacements $P\left(\log _{10}(\delta r) ; t\right)$ for the B particles. Wider lines indicates increased time $t$. (a): $T=0.5 .0$ for $t=0.01,0.05,0.1$, and 0.5 listed from left to right. (b): $T=0.60$ for $t=1,5,10$, 15, 20 and 25 listed from left to right. (c): $T=0.47$ for $t=100,200,300,400$, and 500 listed from left to right. (d): $T=0.44$ for $t=600,700,800,1000,3000,4000$, and 5000 listed from left to right.

weights very strongly the particles which have not moved as far as expected from a Gaussian distribution of particles displacements through the term $\left\langle 1 / \delta r^{2}\right\rangle$ and weights the particles which move farther than expected from a Gaussian distribution through the $\left\langle\delta r^{2}\right\rangle$ term. The factor of $1 / 3$ ensures that $\gamma(t)$ is zero when the self part of the van Hove correlation function is Gaussian.

Shown in Fig. 9 is $\gamma(t)$ for the A and B particles. At short times, the motion of the particles are Gaussian and $\gamma(t)$ is close to zero. At intermediate times there is a peak in $\gamma(t)$ whose height and position increases with decreasing temperature. The peak in $\gamma(t)$ is larger for the $\mathrm{B}$ particles, which is what we expect from examining the self part of the van Hove correlation function and $P\left(\log _{10}(\delta r) ; t\right)$. At long times $\gamma(t)$ decays to zero.

It is interesting to note the temperature dependence of the the average mean square displacement at the peak position of $\gamma(t)$ : for $T \geq 0.8$ the average mean square displacement is independent of temperature and it is around $0.23 \sigma_{A A}^{2}$ for the A particles and $0.31 \sigma_{A A}^{2}$ for the B particles. For $T<0.8$, the average mean squared displacement at the peak position of $\gamma(t)$ increases with decreasing temperature and at $T=0.44$ it reaches the values of approximately $0.84 \sigma_{A A}^{2}$ for the A particles and $2.40 \sigma_{A A}^{2}$ for the $\mathrm{B}$ particles. It should be pointed out that the temperature at which the average mean squared displacement at the peak position of $\gamma(t)$ starts increasing is close to the so-called onset temperature identified for the KobAndersen model by Brumer and Reichman [27].

The insert to each graph in Fig. 9 is the peak position of the new non-Gaussian parameter, $\gamma(t)$, denoted by $\tau_{n n g}$ compared to the $\alpha$ relaxation time and the peak position of the commonly used non-Gaussian parameter, $\alpha_{2}(t)$, denoted by $\tau_{n g}$. Notice that the peak position of $\gamma(t)$ is always greater than the $\alpha$ relaxation time, but it has the same temperature dependence. This is in contrast to $\tau_{n g}$ which is greater than the $\alpha$ relaxation time and equal to $\tau_{n n g}$ at higher temperatures, but increases slower with decreasing temperature than $\tau_{\alpha}$ and $\tau_{n n g}$. For the lowest temperatures, $\tau_{n g}$ is much smaller than either $\tau_{\alpha}$ and $\tau_{n n g}$.

The peak position approximately corresponds to the time in which the two peaks of $P\left(\log _{10}(\delta r) ; t\right)$ are of equal height. Shown in Fig. [10]and Fig.[11] is the $P\left(\log _{10}(\delta r) ; t\right)$ for the A and B particles at the peak position of $\alpha_{2}(t)$ (dashed lines) and $\gamma(t)$ (solid lines) for temperatures of $T=0.47$ and $T=0.44$. Notice that at the time corresponding to the peak position of $\alpha_{2}(t)$, there is at most a shoulder in $P\left(\log _{10}(\delta r) ; t\right)$. For the time which corresponds to the position of the peak in $\gamma(t)$, the two peaks 

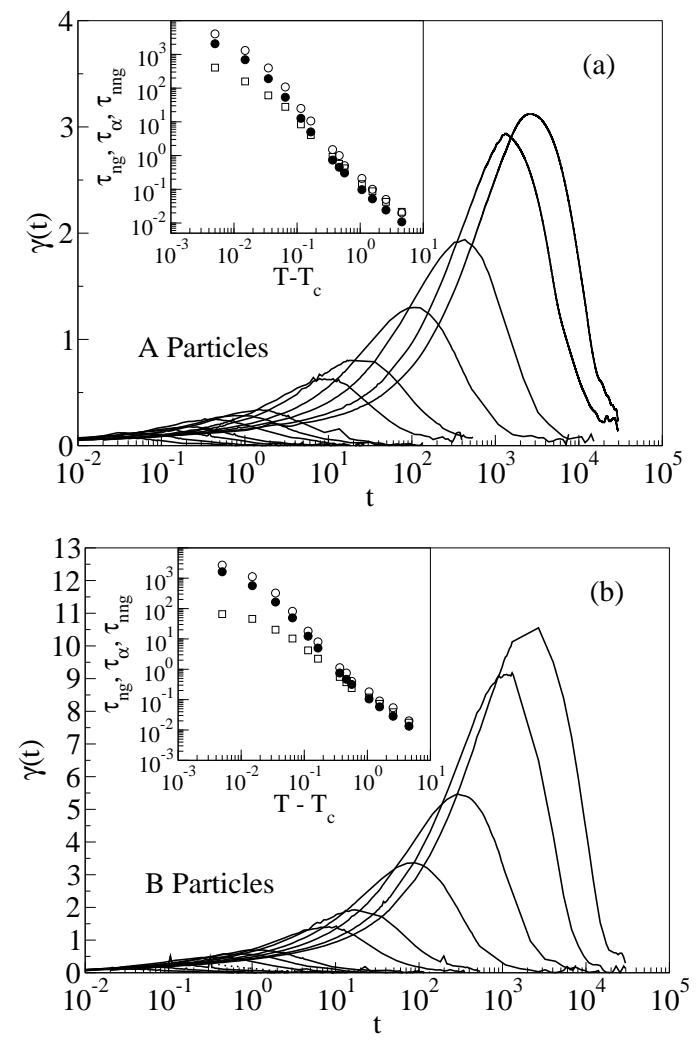

FIG. 9: The new non-Gaussian parameter $\gamma(t)$ for $T=5.0$, $3.0,2.0,1.5,1.0,0.9,0.8,0.6,0.55,0.50,0.47,0.45,0.44$ for the A (a) and B (b) particles. The insets are the position of the peak of $\gamma(t)$, (open circles) compared to $\alpha$ relaxation time (closed circles) and the peak position of $\alpha_{2}(t)$ (squares) versus temperature.

in $P(\log 10(\delta r) ; t)$ are of similar height and the definition of mobile and immobile particles is clear. For comparison, the self part of the van Hove correlation function at the peak position of $\alpha_{2}(t)$ (dashed lines) and $\gamma(t)$ (solid lines) are shown as inserts in Fig. 10] and Fig. 11]

\section{CONCLUSIONS}

The mode-coupling theory has been used extensively to describe the relaxation in supercooled liquids and the glass transition. It correctly describes many qualitative features of the glass transitions observed experimentally and in computer simulations. The most notable success of the mode-coupling theory is that it correctly describes the two step decay of the intermediate scattering functions and the qualitative features of the mean square displacement as a function of time.

The idealized mode-coupling theory predicts power law divergence of the $\alpha$ relaxation time and power law vanishing of the self-diffusion coefficient at a transition temperature $T_{c}$. It has been observed that there is power law like behavior of the $\alpha$ relaxation time and the diffusion coefficient in simulations and experiments close to a
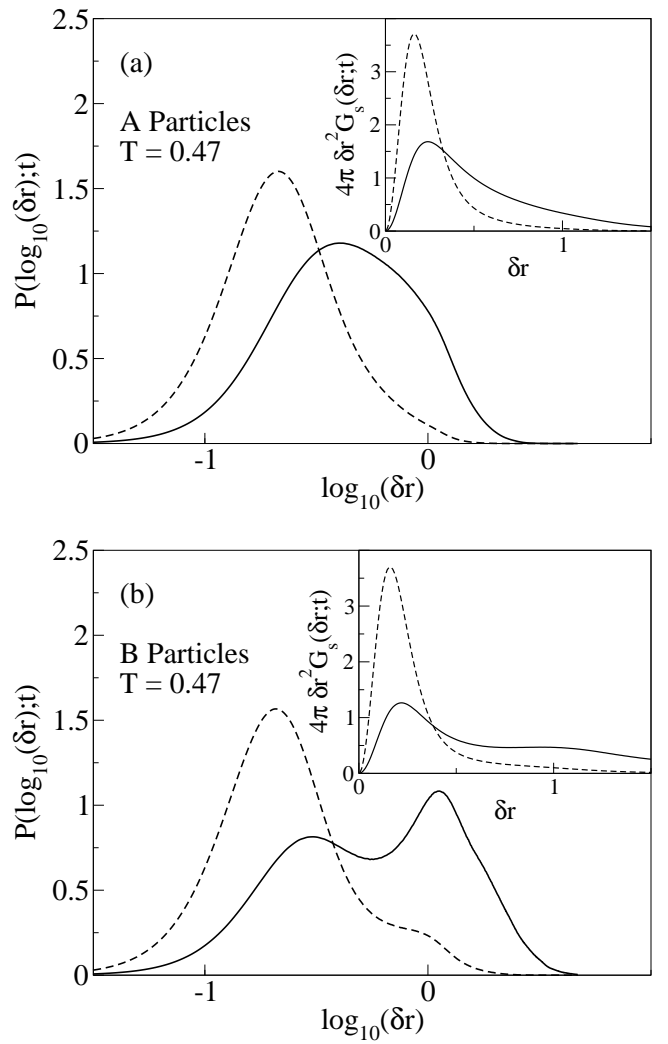

FIG. 10: The probability of the logarithm of the displacements at the the peak position of the the old non-Gaussian parameter $\alpha_{2}(t)$ (dashed line) and the new non-Gaussian parameter $\gamma(t)$ (solid line) at $T=0.47$ for the A particles (a) and the B particles (b). The inserts are the self part of the van Hove correlation functions at the peak position of $\alpha_{2}(t)$ (dashed line) and $\gamma(t)$ (solid line).

temperature $T_{c}$. However, the structural arrest predicted by the mode-coupling theory does not occur at $T_{c}$, but rather there appears to be a crossover to a different relaxation scenario and the mode-coupling transition is said to be "avoided".

We performed Brownian dynamics simulations of a frequently studied glass forming binary mixture for a large range of temperatures. The temperature dependence of the $\alpha$ relaxation time and the diffusion coefficient were similar to what was observed in previous simulations of the same system 1, 3, 41. We found that the diffusion coefficient and the $\alpha$ relaxation time can also be fit to a power law where the transition temperature is $T_{c}^{(2)}=$ 0.401 which is lower than the generally accepted modecoupling transition temperature $T_{c}=0.435$. However, by examining the probability distributions of the logarithm of single particle displacements, $P\left(\log _{10}(\delta r) ; t\right)$, we demonstrated that the crossover from the high temperature diffusive relaxation of the particles to low temperature hopping-like motion occurs near the generally accepted mode-coupling transition temperature $T_{c}=0.435$.

The change in the relaxation processes are evident when one examines the distribution of the logarithm of 

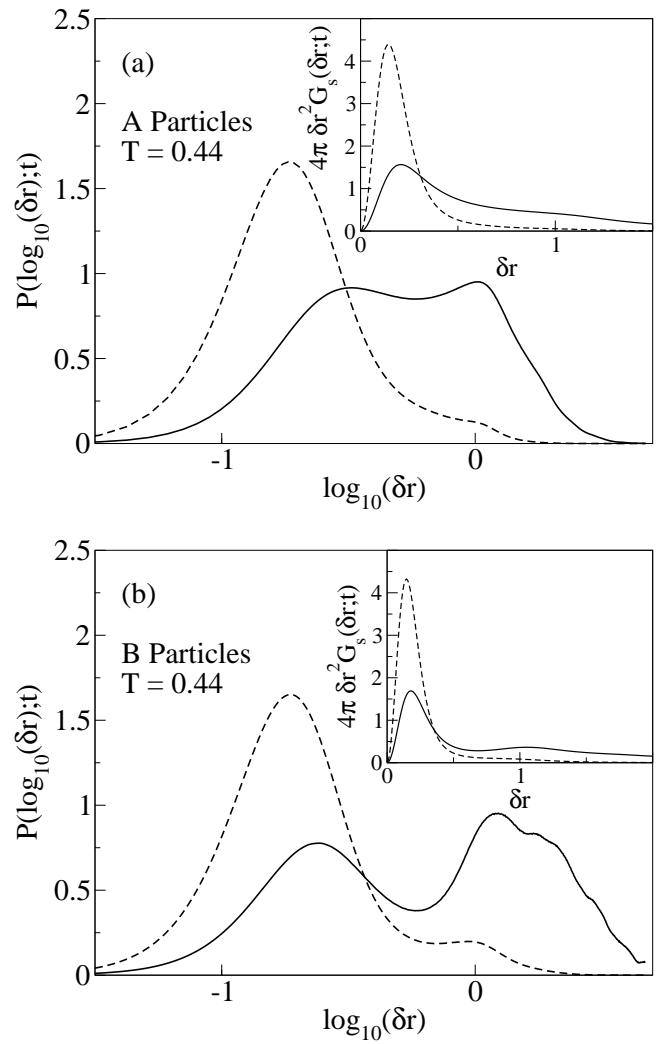

FIG. 11: The probability of the logarithm of the displacements at the peak position of the old non-Gaussian parameter $\alpha_{2}(t)$ (dashed line) and the new non-Gaussian parameter $\gamma(t)$ (solid line) for $T=0.44$ for the A particles (a) and the B particles (b). The inserts are the self part of the van Hove correlation functions at the peak position of $\alpha_{2}(t)$ (dashed line) and $\gamma(t)$ (solid line).

single particle displacements $P\left(\log _{10}(\delta r) ; t\right)$. At higher temperatures there is one peak in $P\left(\log _{10}(\delta r) ; t\right)$ whose position increases for increased time. For lower temperatures, the distribution becomes broad at a time scale right after the plateau region of the mean square displacement. At the lowest temperatures examined in this study, there are two distinct peaks in $P\left(\log _{10}(\delta r) ; t\right)$ for both the A and B particles. The two peaks are evidence that on an intermediate time scale the particles can be separated by their individual relaxation time, and thus are dynamically heterogeneous. Moreover, the minimum between the peaks is smaller for the B particles than for the A particles at a fixed temperature. The dependence on particles size of the probability distributions is similar to what was observed in earlier simulations [14, 23].

One possible interpretation of our results could be that the mode-coupling temperature does not have any physical significance and the crossover in supercooled liquid's dynamics is very smooth 29]. While such an interpretation cannot be excluded, we would like to advocate a more cautious conclusion: in order to identify a crossover temperature (or a narrow crossover temperature range) that one could interpret as mode-coupling temperature, one has to investigate not only power-law fits to transport coefficients and/or relaxation times but also whether microscopic dynamics is homogeneous and diffusive-like or heterogeneous and hopping-like. We would like to emphasize that although the mode-coupling theory, in its standard form, cannot describe hopping-like dynamics, it does provide a reasonable [22] description of dynamics in moderately supercooled fluids.

We found that the typical non-Gaussian parameter $\alpha_{2}(t)$ does a poor job of identifying the time scale on which the heterogeneous, hopping-like motion is most evident. We defined a new non-Gaussian parameter $\gamma(t)=\frac{1}{3}\left\langle\delta r^{2}\right\rangle\left\langle 1 / \delta r^{2}\right\rangle-1$. For temperatures in which there are two peaks in $P\left(\log _{10}(\delta r) ; t\right), \gamma(t)$ has a peak occurring at a time $\tau_{n n g}$ in which the two peaks have approximately the same height. For times in which only one peak is present, the peak position of $\gamma(t)$ identifies the time in which $P\left(\log _{10}(\delta r) ; t\right)$ is widest. The position of the peak for $\alpha_{2}(t)$ and $\gamma(t)$ are the same for high temperatures, but the peak position of $\alpha_{2}(t)$ increases slower with decreasing temperature than the peak position of $\gamma(t)$. Moreover, the peak position of $\gamma(t)$ has the same temperature dependence as the $\alpha$ relaxation time.

\section{Acknowledgments}

We gratefully acknowledge the support of NSF Grant No. CHE 0111152.
[1] W. Kob and H.C. Andersen, Phys. Rev. Lett. 73, 1376 (1994); Phys. Rev. E 51, 4626 (1995); Phys. Rev. E 52, 4134 (1995).

[2] W. Götze, in Liquids, Freezing and the Glass Transition, edited by J.P. Hansen, D. Levesque, and J. Zinn-Justin (North Holland, Amsterdam, 1991).

[3] W. Kob, in Slow Relaxations and Nonequilibrium Dynamics in Condensed Matter, J.L. Barrat et al., eds. (EDP Sciences-Springer Verlag, 2003).

[4] T. Gleim, PhD Dissertation, unpublished.

[5] S. P. Das, Rev. Mod. Phys. 76, 785 (2004).
[6] P. Lunkenheimer, A. Pimenov and A. Loidl, Phys. Rev. Lett. 78, 2995 (1997).

[7] A theoretical description of such a scenario has been proposed by Schweizer and Saltzman; see K.S. Schweizer and E.J. Saltzman, J. Chem. Phys. 119, 1181 (2003), and E.J. Saltzman and K.S. Schweizer, J. Chem. Phys. 119, 1197 (2003).

[8] J.P. Hansen and J.R. McDonald, Theory of Simple Liquids, 2nd ed. (Academic, London, 1986).

[9] See, e.g., J.P. Hansen, Physica A 201, 138 (1995) and references therein. 
[10] S.C. Glotzer, J. Non-Cryst. Solids 247, 342 (2000).

[11] C. Donati et al., Phys. Rev. Lett. 80, 2338 (1998).

[12] W. Kob, C. Donati, S.J. Plimpton, P.H. Poole and S.C. Glotzer, Phys. Rev. Lett. 79, 2827 (1997).

[13] C. Donati, S.C. Glotzer, P.H. Poole, W. Kob and S.J. Plimpton, Phys. Rev. E 60, 3107 (1999).

[14] D.R. Reichman, E. Rabani and P.L. Geissler, cond-mat/0406136

[15] G. Szamel and E. Flenner, Europhys. Lett. 67, 779 (2004).

[16] E. Flenner and G. Szamel, Phys. Rev. E 70, 052501 (2004).

[17] N. Lačević and S.C. Glotzer, J. Phys. Cond. Matt. 15, S2437 (2003); N. Lačević et al., J. Chem. Phys. 119, 7372 (2003).

[18] W. van Megen, T.C. Mortensen, S.R. Williams and J. Müller, Phys. Rev. E 58, 6073 (1998).

[19] T. Gleim, W. Kob and K. Binder, Phys. Rev. Lett. 81, 4404 (1998).

[20] We thank one of the referees of the preliminary report, Ref. [15], for the suggestion.
[21] J.A. Rice, Mathematical Statistics and Data Analysis, (Duxbury Press, 1995).

[22] E. Flenner and G. Szamel, in preparation.

[23] A.M. Puertas, M. Fuchs and M.E. Cates, J. Chem. Phys. 121 (6), 2813 (2004).

[24] M.E. Cates, M. Fuchs, K. Kroy, W.C.K. Poon and A.M. Puertas, cond-mat/0403684

[25] If the van Hove correlation function is Gaussian, all the central moments of the probability distribution of the logarithm of single-particle displacements, $\int_{-\infty}^{\infty}(x-\bar{x})^{n} P(x ; t) \mathrm{d} x$, where $\bar{x}=\int_{-\infty}^{\infty} x P(x ; t) \mathrm{d} x$, are independent of time.

[26] H. Löwen, J.P. Hansen and J.N. Roux, Phys. Rev. A 44, 1169 (1991).

[27] Y. Brumer and D.R. Reichman, Phys. Rev. E 69, 041202 (2004).

[28] M. Fuchs, W. Götze, and M.R. Mayr, Phys. Rev. E 58, 3384 (1998).

[29] L. Berthier and J.P. Garrahan, Phys. Rev. E 68, 041201 (2003). 